\title{
A new Approach for Solving Linear Programming with Grey Variables Problem
}

\author{
Davood Darvishi ${ }^{1, *}$
}

\begin{abstract}
Linear programming problems with interval grey numbers have recently attracted some interest. In this paper, we study linear programs in which right hand sides are interval grey numbers. This model is relevant when uncertain and inaccurate factors make difficult the assignment of a single value to each right hand side. Some methods have been developed for solving these problems. In this paper, we propose a new approach for solving interval grey number linear programming problems is introduced without converting them to classical linear programming problems. Numerical example is provided to illustrate the proposed approach.
\end{abstract}

Keywords: Grey number, Grey linear programming, Optimization, uncertainty.

Manuscript was received on 01/10/2017, revised on 04/05/2018 and accepted for publication on 18/06/2018.

\section{Introduction}

Many application problems, modeled as mathematical programming problems, may be formulated with uncertainty. Grey systems theory is one of the methods used to study problems involving uncertainty. Grey systems theory has been used in many fields, such as economics, management, agricultural, industrial, engineering, health care and assignment, because of its unique characteristics. Most of real world processes existing in decision problems are in a grey stage due to lack of information and uncertainty. This is the reason why the theory of grey optimization provides a useful tool for decision making problems under such uncertainties. The scientific fields covered by grey systems theory include systems analysis, data processing, modeling, prediction, decisionmaking and control. Situations studied in these fields are neither deterministic nor totally unknown, but rather they are partially known. Linear programming problems with imprecise decision variables or parameters play major roles in many applications in various areas. By combining the grey theory with the principle and method of linear programming problem, the linear programming model is established based on the grey theory [15]. Grey programming approach is useful to treat a problem under uncertainty. Grey linear programming is a model of grey systems analysis for decision making under uncertainty. It is an advanced development of the traditional linear programming method. Grey programming has the ability to deal with poor, incomplete, or uncertainty problems with respect to systems, and it has been widely used in many disciplines, such as economics, agriculture, medicine, geography, industry, etc. [10]. Grey programming is different from interval programming. The main difference of grey programming and interval programming is the concept of grey parameter and interval parameter. Grey parameter is a parameter which belongs to an interval, but the interval parameter is a set in the form of an interval [15]. Chen et al. [3] used mathematical programming problems with grey interval numbers and developed grey forecasting. Xia et al. [19] proposed a grey general data envelopment analysis model based on grey linear

\footnotetext{
* Corresponding Author.

${ }^{1}$ Department of Mathematics, Payame Noor University, Tehran, Iran. Email: d_darvishi@ @nu.ac.ir.
} 
programming. They produced the optimal solution of their grey general data envelopment analysis by using a method of $\theta$-positioned programming method. Several methods for solving grey linear programming problems can be seen in [2, 9, 11, 15]. Mokhtari [14] presented a single machine capacitated production planning problem by employing a linear programming formulation. The production costs were assumed to be uncertain parameters. To handle the uncertainties in the model, grey systems theory was employed and the concept of grey numbers was incorporated into an optimization framework. The framework stands for a development of the classical linear programming which allows uncertainty to be effectively communicated into the optimization process. Finally, the uncertain problem was transformed into a grey linear programming, and solved by applying two linear deterministic sub-models. Grey numbers can handle the uncertainty in projects without requiring a predefined membership or probability function. In this paper, we propose a new approach for solving interval grey number linear programming problems is introduced without converting them to classical linear programming problems. The results will be also useful for post optimality analysis.

Our discussion here is outlined as follows: Some necessary backgrounds and definitions of the grey systems theory, grey numbers and grey order relations are presented in Section 2. A definition of the grey linear programming with interval grey numbers right hand side problem is given in Section 3. Furthermore, the fundamental concepts and theorems are given to complete our discussion in this section. In Section 4, numerical examples are modeled and solved by the proposed procedure. Finally, Section 5 consists of discussion and conclusions.

\section{Preliminaries}

In this section, some definitions, concepts, notions and results which are useful in our further consideration about grey system theory and in particular, for the interval grey numbers calculus as a tool for solving the grey linear programming, is introduced [8, 12,13]. A grey system is defined as a system that involves non-deterministic information.

As a different model for uncertainty representation, professor Deng proposed grey systems theory in 1982 [5]. The grey systems theory focuses on the study of such uncertain systems with partially known and partially unknown information whereby matters of the characteristics of poor information are seen as its research subjects [7]. Grey system theory is set of methods used to interpret uncertainty of parameters that works well for mathematical analysis of systems with uncertain information. During 2000-2015, it gradually moves toward a mature period for grey systems theory to grow from adolescence to young excessive. In the meantime, the grey systems theory has formed a theoretical structure that is generally accepted, and become a course at many universities all around the world [13].

Definition 2.1. A grey system is defined as a system containing uncertain information presented by a grey number and grey variables.

Grey system theory introduces the concept of interval grey numbers. Grey numbers are regarded as the basic unit of grey systems to participate in the construction of grey model. Grey number is an important concept in grey systems theory. Grey numbers find use in optimization to proactively incorporate uncertainties expressed as intervals and identify maximal, stable, feasible ranges for the objective function and decision variables [17].

Definition 2.2. An interval grey number is a number with clear upper and lower boundaries, but which has an unclear position within the boundaries.

An interval grey number is expressed mathematically as: 


$$
\otimes x \in[\underline{x}, \bar{x}]=\{\underline{x} \leq t \leq \bar{x}\}
$$

where $\otimes x$ is a grey number, $t$, is information, $\underline{x}$ and $\bar{x}$ are the lower and upper limits of the information. In addition, the lower and upper bounds of this number cannot be equal [4].

There are several types of grey numbers [12] among them the interval grey numbers are considered here as the convenient kind in the literature.

Remark 2.1. We denote the set of all grey numbers by $R(\otimes)$. We also show an element of $R(\otimes)$, that is $\otimes x \in[\underline{x}, \bar{x}]$ by $[\underline{x}, \bar{x}]_{G}$.

The main arithmetic operations can be defined on grey numbers. Let $\otimes x_{1} \in\left[\underline{x}_{1}, \bar{x}_{1}\right]$ and $\otimes x_{2} \in\left[\underline{x}_{2}, \bar{x}_{2}\right]$ be two grey numbers. The following operations can be defined [12]:

$\otimes x_{1}+\otimes x_{2}=\left[\underline{x}_{1}+\underline{x}_{2}, \bar{x}_{1}+\bar{x}_{2}\right]$

$\otimes x_{1}-\otimes x_{2}=\left[\underline{x}_{1}-\bar{x}_{2}, \bar{x}_{1}-\underline{x}_{2}\right]$

$\otimes x_{1} \times \otimes x_{2}=\left[\min \left(\underline{x}_{1} \underline{x}_{2}, \underline{x}_{1} \bar{x}_{2}, \bar{x}_{1} \underline{x}_{2}, \bar{x}_{1} \bar{x}_{2}\right), \max \left(\underline{x}_{1} \underline{x}_{2}, \underline{x}_{1} \bar{x}_{2}, \bar{x}_{1} \underline{x}_{2}, \bar{x}_{1} \bar{x}_{2}\right)\right]$

$\otimes x_{1} \div \otimes x_{2}=\left[\underline{x}_{1}, \bar{x}_{1}\right] \times\left[\frac{1}{\bar{x}_{2}}, \frac{1}{\underline{x}_{2}}\right]$, where $0 \notin\left[\underline{x}_{2}, \bar{x}_{2}\right]$.

Lemma 2.1. If $k \in R-\{0\}, \otimes x \in[\underline{x}, \bar{x}]$ be a grey number, then we have:

$$
\begin{gathered}
k .(\otimes x)=\otimes(k x) \in[k \underline{x}, k \bar{x}], \text { if } k>0, \\
k .(\otimes x)=\otimes(k x) \in[k \bar{x}, k \underline{x}], \text { if } k<0 .
\end{gathered}
$$

Definition 2.3. A whitening value of a grey number $\otimes x$, is defined as a deterministic number in which its value is lying between the upper and lower bounds of $\otimes x$ (see in [20])

$$
\underline{x} \leq \tilde{\otimes} x \leq \bar{x}
$$

where $\tilde{\otimes} x$ is a whitening value of $\otimes x$. This relation can be expressed as:

$$
\tilde{\otimes} x=\underline{x}+\alpha(\bar{x}-\underline{x})
$$

Remark 2.2. For any real number $a$, we say $\otimes a$ is corresponding grey number, if $\otimes a=[a, a]$. In fact, any real number $a$ is a white number. Thus without loss of generality, throughout the paper we let $\otimes 0=[0,0]$ as the zero grey number and for the sake of convenience referred them as $\otimes 0$ or 0 .

There are several approaches to rank the grey numbers. Grey number is the basic unit of grey system and the operations of grey numbers are different from regular interval numbers.

Definition 2.4. Let $\otimes \hat{x}$ and $g^{\circ}(\otimes x)$ be respectively the kernel and the degree of greyness of a grey number $\otimes x$. Then, $\otimes x=\otimes \hat{x}_{\left(g^{\circ}\right)}$ is seen as a simple form of the grey number $\otimes x$.

Definition 2.5. Suppose $\otimes x$ and $\otimes y$ are two grey numbers and $\otimes \hat{x}, \otimes \hat{y}$ are the kernel of $\otimes x$ and $\otimes y$, respectively, $g^{\circ}(\otimes x)$ and $g^{\circ}(\otimes y)$ are the degree of greyness of $\otimes x$ and $\otimes y$, respectively. So, 
if $\otimes \hat{x}<\otimes \hat{y}$, thus $\otimes x<_{G} \otimes y$;

if $\otimes \hat{x}=\otimes \hat{y}$, thus

(i) if $g^{\circ}(\otimes x)=g^{\circ}(\otimes y)$, thus $\otimes x={ }_{G} \otimes y$;

(ii) if $g^{\circ}(\otimes x)<g^{\circ}(\otimes y)$, thus $\otimes x>_{G} \otimes y$;

(iii) if $g^{\circ}(\otimes x)>g^{\circ}(\otimes y)$, thus $\otimes x<_{G} \otimes y$.

\section{Grey Linear Programming}

Consider a diet problem that involves choosing a combination of foods that meet the minimum nutritional needs of a diet. We formulate this as follows:

$$
\begin{aligned}
& \text { Min } \mathrm{Z}=\mathrm{Cx} \\
& \text { s.t. } A x \geq b \\
& x \geq 0
\end{aligned}
$$

Suppose a pharmaceutical company produces each of the basic nutrients in the form of a diet pill. The pharmaceutical company is trying to convince people who are on a diet to buy these pills and provide the necessary food directly instead of buying different foods. The problem for the pharmaceutical company is to determine the positive prices of each unit of food pills $\mathrm{v}_{1}, \mathrm{v}_{2}, \ldots, \mathrm{v}_{\mathrm{m}}$. So that the company's income is maximized and while these pills are competitive with real food, the cost of a unit of feed, which is artificially prepared from a combination of pure foods, should not be more than $\mathrm{c}_{\mathrm{i}}$, the price of this feed in the market. If unit $b$ is purchased from a food safety entity, the company's problem modeling will be as follows:

$$
\begin{aligned}
\text { Max } & \mathrm{W} & =\mathrm{vb} \\
\text { s.t. } & \mathrm{vA} & \leq \mathrm{c} \\
& \mathrm{v} & \geq 0
\end{aligned}
$$

Now, if there is no accurate data on such issues, or if obtaining sufficient information on the subject is costly, then uncertainty approaches should be used. The linear programming that involves grey numbers has been called grey linear programming. Grey linear programming is an approach developed for decision making under uncertainty. It is a further development of the traditional linear programming method. Deng $[6,7]$ developed the grey systems theory and presented several grey decision-making systems. Grey numbers have been applied to a variety of programs with applications including hypothetical numerical examples of solid waste management, water resources allocation, optimized urban water supply scheme, chemical integrated system, buildings deformation monitoring and web service selection. Grey programming has the ability to deal with poor, incomplete, or uncertainty problems with systems, and it has been widely used in many aspects such as economics, agriculture, medicine, geography, industry, etc. [10]. Several types of uncertainties are encountered in the design of machines and structures. Razavi et al. [16] proposed a multi objective model to solve grey linear programming problems in general form. The suggested approach is mainly based on the concept of order relations between grey numbers. Based on this notion, a modeling process is proposed to convert the original grey linear programming problem to a bi objective problem. Baidya et al. [1] proposed two new multi-objective multi-stage solid transportation problems under grey uncertainty where unit transportation cost, time, purchasing costs, supplies, demands, conveyances capacities were considered as interval grey numbers. In a 
grey linear programming problem, variables or parameters or a combination of them are considered to be grey numbers.

In this section, we define variables of linear programming are grey numbers, naming it as grey variables linear programming problem.

Grey variables linear programming problem as follow:

$$
\begin{aligned}
& \text { Max } \otimes Z={ }_{G} \sum_{j=1}^{n} c_{j} \otimes x_{j} \\
& \text { subject to } \sum_{j=1}^{n} a_{i j} \otimes x_{j} \leq_{G} \otimes b_{i}, \quad i=1,2,3, \ldots, m, \\
& \otimes x_{j} \geq_{G} \otimes 0, j=1,2, \ldots, n .
\end{aligned}
$$

where $\otimes b \in R(\otimes)^{m}, A \in R^{m \times n}$ and $c \in R^{n}$ are given and $\otimes X \in R(\otimes)^{n}$ is to be determined. In this model, the coefficient matrix, $\mathrm{A}$, is crisp, but the decision vector $\otimes X$ is composed of grey numbers.

Note that a grey variables linear programming problem is a linear programming problem in grey environment in which the decision making variables and right-hand sides are interval grey numbers. Now, we may rewrite (1) as follows:

$$
\begin{gathered}
\max \otimes z={ }_{G} c \otimes x+0 \otimes y \\
\text { s.t. } A \otimes x+\otimes y={ }_{G} \otimes b \\
\otimes x, \otimes y \geq_{G} \otimes 0,
\end{gathered}
$$

where $\otimes y=\left(\otimes y_{1}, \otimes y_{2}, \ldots, \otimes y_{n}\right)$.

Define $\bar{x} \in R(\otimes)^{n+m}$ and $\bar{c} \in \mathbb{R}^{n+m}$ as

$$
\begin{aligned}
& \bar{x}_{j}=\left\{\begin{array}{cc}
\otimes x_{j} & j=1,2,3, \ldots, n, \\
\otimes y_{j-n} & j=n+1, n+2, \ldots, n+m
\end{array}\right. \\
& \bar{c}_{j}= \begin{cases}c_{j} & j=1,2,3, \ldots, n, \\
0 & j=n+1, n+2, \ldots, n+m\end{cases}
\end{aligned}
$$

Suppose that a basic solution for (2) is given by $\bar{x}_{B}={ }_{G} B^{-1} \otimes b$, with the basis matrix $B$. Now, let $z_{j}=\bar{c}_{B} B^{-1} \bar{a}_{j}, \otimes y_{0}={ }_{G} B^{-1} \otimes b$, where $\bar{c}_{B}=\left(\bar{c}_{B_{1}}, \bar{c}_{B_{2}}, \ldots, \bar{c}_{B_{m}}\right)$ and $\bar{a}_{j}$ is the $j$ th column of the coefficient matrix [A, I].

Consider Table 1 , where $\left(\bar{x}_{B}\right)_{r}$ is the $r$ th grey basic variable, $y_{j}=B^{-1} \bar{a}_{j}$. Suppose that for $j=1,2,3, \ldots, n+m$, we have

$$
z_{j}-\bar{c}_{j} \leq 0
$$

Define $w=\bar{c}_{B} B^{-1}$, where $w=\left(w_{1}, w_{2}, \ldots, w_{m}\right)$. For $j=1,2,3, \ldots, n$, we have

$$
y_{0 j}=z_{j}-\bar{c}_{j}=\bar{c}_{B} B^{-1} a_{j}-c_{j}=w a_{j}-c_{j} .
$$

Therefore, for $j=1,2,3, \ldots, n$, from $z_{j}-c_{j} \leq 0$, it follows that $w a_{j}-c_{j} \leq 0$. then, $w A \leq c$.

On the other hand, using (3), we have,

$$
0 \geq z_{n+i}-\bar{c}_{n+i}=c_{B} B^{-1} e_{i}-0=w e_{i}=w_{i}, i=1,2, \ldots, m,
$$


and hence, $w \leq 0$ yielding the dual feasibility. If $\otimes y_{r 0} \geq 0 \otimes$, for all $r=1, \ldots, m$, then a grey feasible solution for the grey variable linear programming problem is at hand. Moreover, we will have,

$$
\overline{c x}={ }_{G} \bar{c}_{B} \otimes y_{0}={ }_{G} \bar{c}_{B} B^{-1} \otimes b={ }_{G} w \otimes b
$$

and thus, establishing the optimality of $\bar{x}$ and $w$ for the grey variable linear programming and dual grey variable linear programming problems, respectively. Therefore, we have the following result.

Corollary3.1. The optimality criteria $z_{j}-\bar{c}_{j} \leq 0$ for all $j$, for the grey variable linear programming problem is equivalent to the feasibility condition for the dual grey variable linear programming problem. If, in addition, $\bar{x}$ corresponding to a basis $B$ is primal feasible then $\bar{x}$ is optimal for the grey variable linear programming problem and $w=\bar{c}_{B} B^{-1}$ is optimal to the dual grey variable linear programming problem.

Now, let's assume that the dual grey variable linear programming problem is feasible and $\bar{x}$, corresponding to a basis $B$, is dual feasible but primal infeasible (and hence not optimal). That is, we have,

$$
y_{0 j}=z_{j}-\bar{c}_{j} \leq 0, j=1,2, \ldots, n+m
$$

and there exists at least one $r$ such that $\otimes y_{r 0} \leq_{G} \otimes 0$. We can immediately deduce that the primal problem must be finite.

Thus, the grey variable linear programming problem can be either infeasible (in which case, the dual grey variable linear programming problem is unbounded) or it has an optimal solution. In what follows we will show how to work on row $r$ of the simplex tableau corresponding to $B$, as the pivoting row and either

1) Detect infeasibility of the grey variable linear programming problem (or unboundedness of the dual grey variable linear programming problem) or

2) Find a column $l$, as the pivoting column, to pivot on $y_{r l}$ and obtain a new dual feasible tableau with a no decreasing primal objective value.

Table 1. A dual feasible simplex tableau

\begin{tabular}{lcccccc}
\hline Basis & $x_{1}$ & $\cdots$ & $x_{l}$ & $\cdots$ & $x_{n+m}$ & R.H.S. \\
$Z$ & $z_{1}-c_{1}$ & $\cdots$ & $z_{l}-c_{l}$ & $\cdots$ & $z_{n+m}-c_{n+m}$ & $c_{B} y_{0}$ \\
\hline$\left(x_{B}\right)_{1}$ & $y_{11}$ & $\cdots$ & $y_{1 l}$ & $\cdots$ & $y_{1, n+m}$ & $y_{10}$ \\
$\vdots$ & $\vdots$ & & $\vdots$ & & $\vdots$ & $\vdots$ \\
$\left(x_{B}\right)_{r}$ & $y_{r 1}$ & $\cdots$ & $y_{r l}$ & $\cdots$ & $y_{r, n+m}$ & $y_{r 0}$ \\
$\vdots$ & $\vdots$ & & $\vdots$ & & $\vdots$ & $\vdots$ \\
$\left(x_{B}\right)_{m}$ & $y_{m 1}$ & $\cdots$ & $y_{m l}$ & $\cdots$ & $y_{m, n+m}$ & $y_{m 0}$ \\
\hline
\end{tabular}

Lemma3.1. If in a dual feasible simplex tableau an $r$ exists such that $\otimes y_{r 0}<_{G} \otimes 0$ and $y_{r j} \geq 0$, for all $j$, then the grey variable linear programming problem is infeasible. 
Proof. Suppose that a grey dual simplex tableau is feasible and an $r$ exists such that $\otimes y_{r 0}<_{G} \otimes 0$ and $y_{r j} \geq 0$, for all $j$. Corresponding to the $r$ th row of the tableau, we have $\sum_{j} y_{r j} \bar{x}_{j}={ }_{G} \otimes y_{r 0}$.

Since $y_{r j} \geq 0$ for all $j$ and $\bar{x}_{j}$ is required to be nonnegative, then $\sum_{j} y_{r j} \bar{x}_{j} \geq_{G} \otimes 0$. for any grey basic feasible solution. However, $\otimes y_{r 0}<_{G} \otimes 0$. This shows that grey variable linear programming problem is infeasiblem.

Lemma3.2. If in a dual feasible simplex tableau, an $r$ exists such that $\otimes y_{r 0}<_{G} \otimes 0$ and there exists a non-basic index $j$ such that $y_{r j}<0$, then a pivoting column $l$ can be found so that pivoting on $y_{r l}$ will yield a dual feasible tableau with a corresponding non-decreasing objective value.

Proof. It is straight forward based on the classical concept of the linear programming.

Now, using the above results, we introduce a new dual algorithm to solve the grey variable linear programming problem directly, making use of the dual feasible simplex tableau. Thus, we refer to the new algorithm as a dual simplex method.

\section{Algorithm 1. A Grey Dual Simplex Method}

1) Dual feasibility: Given a basis $B$ for the grey variable linear programming problem such that $y_{0 j}=z_{j}-\bar{c}_{j} \leq 0$ for all $j$. Compute the simplex tableau.

2) If $\otimes y_{0} \geq_{G} \otimes 0$, then Stop (the current solution is optimal) else select the pivot row $r$ with $\otimes y_{r 0}<_{G} \otimes 0$.

3) If $y_{i j} \geq 0$ for all $j$ then Stop (grey variable linear programming problem is infeasible) else selects the pivot column $l$ by the following minimum ratio test:

4) Pivot on $y_{r l}$ and go to (2).

$$
\frac{y_{0 l}}{y_{r l}}=\min _{j \neq B_{i}}\left\{\frac{y_{0 j}}{y_{r j}} \mid y_{r j}<0\right\}
$$

\section{Numerical Examples}

In this section, for an illustration of the above approach, a numerical example of the linear programming with grey variables problem will be solved based on the proposed method.

Example4.1. The objective of the animal diet problem is to select a family of foods that will satisfy a set of daily nutritional requirements at a minimum cost. The classical diet problem is stated as a linear optimization problem. In grey diet problems with a grey objective in which, for some foods $j=1, \ldots, n$, there is some vagueness on its corresponding cost and a series of constraints to supply adequate nutrients which are modeled by means of grey numbers. With regard to the mathematical version of this problem, we need to define some variables. 
Let us consider a set of foods $f=\left\{x_{1}, x_{2}, \ldots, x_{n}\right\}$, a set of nutrients $N=\left\{A_{1}, A_{2}, \ldots, A_{n}\right\}$, and the following variables:

$\otimes Z$ : Objective function,

$\otimes c_{j}:$ Cost of the food $j, j=1,2, \ldots, n$,

$\otimes x_{j}$ : Amount of food $j$ to eat, $j=1,2, \ldots, n$,

$a_{i j}$ : Amount of nutrient $i$ in food $j, j=1,2, \ldots, n, i=1,2, \ldots, m$,

$b_{i}$ : Amount of nutrient $i$ required, $i=1,2, \ldots, m$,

$i$ : Index identifying feed nutrient components with $i=1,2, \ldots, m$,

$j$ : Index identifying feed components with $j=1,2, \ldots, n$.

A grey linear programming problem covers an objective function and a series of constraints. Usually the objective function classification with $n$ function $\otimes Z\left(x_{1}, x_{2}, \ldots, x_{n}\right)$ in which the optimum amount of classification is as a set of constraints defined by the given limitation with the function of $g_{i}=\left(x_{1}, x_{2}, \ldots, x_{n}\right) \geq(\leq) b_{i}, i=1, \ldots, m$. were determined. Consider the following grey linear programming:

$$
\begin{aligned}
& \min \otimes Z={ }_{G} \sum_{j=1}^{n} \otimes c_{j} \otimes x_{j} \\
& \text { st. } \sum_{j=1}^{n} a_{i j} \otimes x_{j} \geq_{G}(\leq,=) b_{i}, i=1, \ldots, m . \\
& \otimes x_{j} \geq 0, j=1, \ldots, n .
\end{aligned}
$$

The purpose of the designed model is provision of a minimum supply of nutrients by taking into consideration the animal with combined oral specific nutrients which are used to achieve maximum milk production in livestock.

Some feed stuffs including alfalfa hay, barley grain, corn silage, cottonseed meal and sunflower meal were used for the formulation of a ration for Holstein dairy cows. Chemical compositions of

\begin{tabular}{|c|c|c|c|c|c|}
\hline \multirow{2}{*}{ Feeds } & $\mathrm{NEl}$ & Fat & $\mathrm{Ca}$ & $\mathrm{P}$ & Price \\
\hline & (Kcal/kg) & $(\mathrm{g} / \mathrm{kg})$ & $(\mathrm{g} / \mathrm{kg})$ & $(\mathrm{g} / \mathrm{kg})$ & (Rial/kg) \\
\hline Alfalfa & 1200 & 20 & 14 & 3 & 1350014500 \\
\hline Barley grain & 1850 & 20 & 15 & 4 & 80008500 \\
\hline Corn silage & 1400 & 30 & 2.5 & 3 & 20003000 \\
\hline Cottonseed meal & 1650 & 15 & 1.5 & 12 & 1650017500 \\
\hline Sunflower meal & 1350 & 10 & 4.5 & 11 & 1150012500 \\
\hline
\end{tabular}
feeds are presented in Table 2.

Table 2. Chemical compositions of foods

Diets were formulated to supply adequate supply of four nutrients (energy, fat, calcium, phosphorus) for a cow. For example, energy for lactation is reported in kilo calories (kcal) and specifies the amount of energy a dairy cow needs for maintenance and lactation. The maximum and minimal requirements of the foods price as grey numbers. Table 3 shows the nutrient requirements. 
Table 3. The nutrient requirements and their limitation in cows diet

\begin{tabular}{lcc}
\hline Nutrient requirements & Unit & Value \\
\hline Energy & Kcal/kg & 1600 \\
Ether extract & Gram & 70 \\
Calcium $(\mathrm{Ca})$ & Gram & 10 \\
Phosphor (P) & Gram & 5 \\
Alfalfa hay & Gram & 220 \\
Barley grain & Gram & 280 \\
Corn silage & Gram & 100 \\
Cottonseed meal & Gram & 100 \\
Sunflower meal & Gram & 80 \\
\hline
\end{tabular}

We establish the minimized cost grey diet problem model as follows:

$$
\begin{aligned}
& \min \otimes Z=\otimes[13500,14500] \otimes x_{1}+\otimes[8000,8500] \otimes x_{2}+\otimes[2000,3000] \otimes x_{3}+ \\
& \otimes[16500,17500] \otimes x_{4}+\otimes[11500,12500] \otimes x_{5}
\end{aligned}
$$

All constraints are briefly described as follows:

$$
\begin{aligned}
& \text { s.t. } 1200 \otimes x_{1}+1850 \otimes x_{2}+1400 \otimes x_{3}+150 \otimes x_{4}+1350 \otimes x_{5} \geq 1600 \\
& 20 \otimes x_{1}+20 \otimes x_{2}+30 \otimes x_{3}+15 \otimes x_{4}+10 \otimes x_{5} \geq 70 \\
& 14 \otimes x_{1}+15 \otimes x_{2}+2.5 \otimes x_{3}+1.5 \otimes x_{4}+4.5 \otimes x_{5} \geq 10 \\
& 3 \otimes x_{1}+4 \otimes x_{2}+3 \otimes x_{3}+12 \otimes x_{4}+11 \otimes x_{5} \geq 5 \\
& \otimes x_{1}+\otimes x_{2}+\otimes x_{3}+\otimes x_{4}+\otimes x_{5}=1 \\
& \otimes x_{1} \leq 0.22 \\
& \otimes x_{2} \leq 0.28 \\
& \otimes x_{3} \leq 0.1 \\
& \otimes x_{4} \leq 0.1 \\
& \otimes x_{5} \leq 0.08
\end{aligned}
$$

All the parameters represent their practical significance in the model, and it should be emphasized that all the parameters in the model are nonnegative.

In the mentioned practical problem, it is possible to solve it by using the duality concept of linear programming and by converting the problem into the form of the linear programming with grey variables problem.

Example 4.2. A company wants to know how much to produce from each of its two products to maximize total profits, subject to resource constraints. The labor force and materials needed as well as the profit share of each of the two products are listed in the below Table 4 . 
Table 4. The problem information

\begin{tabular}{lccl}
\hline Sources & Door & window & R.H.S \\
\hline working hours & 3 & 2 & $\otimes[1,5]$ \\
Drawing clock & 4 & 1 & $\otimes[6,8]$ \\
Share of profit per unit & 2 & 3 & \\
\hline
\end{tabular}

The above problem is modeled as follows:

$$
\begin{array}{cc}
\max & \otimes z=2 \otimes x_{1}+3 \otimes x_{2} \\
\text { s.t. } & 3 \otimes x_{1}+2 \otimes x_{2} \leq \otimes[1,5] \\
& 4 \otimes x_{1}+\otimes x_{2} \leq \otimes[6,8] \\
\otimes & x_{1}, \otimes x_{2} \geq \otimes 0
\end{array}
$$

We may first grey simplex tableau as follows:

$$
\begin{array}{cc}
\max \otimes z-2 \otimes x_{1}-3 \otimes x_{2}=\otimes 0 \\
\text { s.t. } \quad 3 \otimes x_{1}+2 \otimes x_{2}+\otimes s_{1}=\otimes[1,5] \\
4 \otimes x_{1}+\otimes x_{2}+\otimes s_{2}=\otimes[6,8] \\
\otimes x_{1}, \otimes x_{2}, \otimes s_{1}, \otimes s_{2} \geq \otimes 0
\end{array}
$$

or equivalently,

Table 5. The first simplex tableau of the primal grey linear programming problem

\begin{tabular}{cccccc}
\hline Basis & $\otimes x_{1}$ & $\otimes x_{2}$ & $\otimes s_{1}$ & $\otimes s_{2}$ & R.H.S. \\
\hline$\otimes Z$ & -2 & -3 & 0 & 0 & $\otimes 0$ \\
$\otimes s_{1}$ & 3 & 2 & 1 & 0 & $\otimes[1,5]$ \\
$\otimes s_{2}$ & 4 & 1 & 0 & 1 & $\otimes[6,8]$ \\
\hline
\end{tabular}

$\otimes s_{1}$ is a leaving variable and $\otimes x_{2}$ is an entering variable. The new tableau is

Table 6. The optimal simplex tableau of the primal grey linear programming problem

\begin{tabular}{cccccc}
\hline Basis & $\otimes x_{1}$ & $\otimes x_{2}$ & $\otimes s_{1}$ & $\otimes s_{2}$ & R.H.S. \\
\hline$\otimes Z$ & 2.5 & 0 & 1.5 & 0 & $\otimes[1.5,7.5]$ \\
$\otimes x_{2}$ & 1.5 & 1 & 0.5 & 0 & $\otimes[0.5,2.5]$ \\
$\otimes s_{2}$ & 0.5 & 0 & -0.5 & 1 & $\otimes[0.5,3.5]$ \\
\hline
\end{tabular}


Since for all $j$, the current basic feasible solution is optimal. The optimal solution is $\left(\otimes x_{1}^{*}, \otimes x_{2}^{*}\right)=(\otimes[0,0], \otimes[0.5,2.5])$ and $\otimes Z^{*}=\otimes[1.5,7.5]$.

\section{Conclusion}

Grey linear programming is a model of grey systems analysis for decision making under uncertainty. An algorithm like well-known primal simplex algorithm for solving grey linear programming problems is proposed, where it can solve the problem without converting to the classical linear programming problems. The proposed approach will be useful for establishing the sensitivity analysis when a real problem. These results would be useful for post optimality analysis and in particular establishing a dual algorithm for solving the dual grey linear programming problem directly on the primal simplex tableau. Future researchers can apply the appropriate model to solve various problems requiring grey linear programming problem. In their future research endeavors, the researchers can aim to propose more accurate methods for solving grey linear and nonlinear models.

\section{Acknowledgement}

The author would like to express his thanks to the valuable comments of the anonymous referees who contributed to improve the earlier version of this manuscript. Also, authors would like to thank the International Center of Optimization and Decision Science (ODM) for its supports.

\section{References}

[1] Baidya, A., Bera, U.K. and Maiti, M. (2016). The grey linear programming approach and its application to multi objective multi stage solid transportation problem, Opsearch, 53(3), 500-522.

[2] Bi, Y.M, Li, J.W and Li, G.M. (2000). Genocop Algorithm for solving grey linear programming problem, System Engineering Theory and Practice, 2, 79-83.

[3] Chen, Z., Chen, Q., Chen, W. and Wang, Y. (2004). Grey linear programming, Kybernetes, 33(2), 238-246.

[4] Cheng, F.J., Hui, S.H. and Chen, Y.C. (2002). Reservoir operation using grey fuzzy stochastic dynamic programming, Hydrological Processes, 16, 2395-2408.

[5] Deng, J.L. (1982). The control problems of grey systems, Systems and Control Letters, 1(5), 288294.

[6] Deng, J.L. (1985). Decision making for grey situations. Fuzzy Mathematics, 5(2), 43-50.

[7] Deng, J.L. (1989). Introduction to grey system theory, The Journal of Grey Theory, 1(1), 1-24.

[8] Li, Q.X. and Lin, Y. (2014). A briefing to grey systems theory, Journal of Systems Science and [8] Information, 2(2), 178-192.

Li, Q.X., Liu S.F. and Wang, N.A. (2014). Covered solution for a grey linear program based on a

[9] general formula for the inverse of a grey matrix. Grey Systems: Theory and Application, 4(1), 7294.

[10] Liang, R.H. (1997). Application of grey linear programming to short term hydro scheduling,

[10] Electric Power Systems Research, 41(3), 159-165.

[11] Liu, S.F., Dang, Y. and Forrest, J. (2009). On positioned solution of linear programming with grey parameters. International Conference on Systems, Man and Cybernetics, USA, 751-756.

[12] Liu, S. F. and Lin, Y. (2006). Grey Information: Theory and Practical Applications, Springer.

[13] Liu, S., Yang, Y., Xie, N. and Forrest, J. (2016). New progress of grey system theory in the new

[13] millennium, Grey Systems: Theory and Application, 6(1), 2-31

[14] Mokhtari, H. (2016). A single machine capacitated production planning problem under uncertainty:

[14] a grey linear programming approach, International Journal of Engineering, 29(4), 524-529. 
Nasseri S.H., Yazdani, A. and Darvishi, D. (2016). A primal simplex algorithm for solving linear [15] programming problem with grey cost coefficients, Journal of New Researches in Mathematics, $1(4), 121-141$.

[16] Razavi, S.H., Akrami, H. and Hashemi, S.S. (2012). A multi objective programming approach to solve grey linear programming, Grey Systems: Theory and Application, 2(2), 259-271.

[17] Rosenberg, D.E. (2009). Shades of grey: a critical review of grey number optimization, Engineering Optimization, 41(6), 573-592.

[18] Weiqin, T. Congjun, R. and Li, L. (2011). Grey linear programming problem of commodities scheduling in large scale emergency, The Journal of Grey System, 3, 281-290.

Xia, D.C., Li, B.T., Wang, J.P. and Shi, G.P. (2014). Research on general data envelopment [19] analysis model based on the grey linear programming, Applied Mechanics and Materials, 577, 828831.

[20] Yang, Y. and John, R. (2012). Grey sets and greyness. Information Sciences, 185, 249-264. 